\title{
Argumentos x Núcleos Focales: El Estatus de Clíticos que Duplican SSNN en Español
}

\author{
(Arguments and Focus Heads: The Status of NP-Doubling \\ Clitics in Spanish) \\ (Argumentos x Núcleos Focais: O Status de Clíticos \\ que Duplicam SNs em Espanhol)
}

\author{
Paulo CORREA \\ (Universidade Federal do Rio de Janeiro)
}

\begin{abstract}
This paper presents a generative analysis of accusative clitics involved in clitic-doubling constructions in Spanish. It will be shown that this kind of clitic does not constitute a single unitary element, but must be classified in two types, the anaphoric one and the deictic one. Whereas the former bave been analyzed in early works such as Jaeggli $(1982,19866)$, the occurrence of the latter may be related to the focalization of a constituent in situ. This analysis parallels with Suner (1995), treating negative concord as a polarity phenomena, once the present work reveals a correlation between the syntactic realization of negative words in negative polarity and the realization of a clitic doubling a focused $N P$.
\end{abstract}

KEY-WORDS: syntax, Spanish, clitic focus.

RESUMO: Este trabalho apresenta uma análise gerativa de clíticos de Caso acusativo envolvidos em construçöes de reduplicação em espanbol. Demonstrará que este tipo de clítico não constitui um elemento único, e, sim, que pode ser dividido em dois grupos, o dos anafóricos e o dos dêiticos. Enquanto os primeiros foram analisados em trabalhos anteriores, como o de Jaeggli (1982, 1986b), a ocorrência dos últimos pode estar vinculada à expressão de um foco in situ. Esta análise encontra um paralelo em Suñer (1995), que trata a concordância negativa como um fenômeno de polaridade, já que este trabalho revela uma correlaşãa entre a realização sintática de palavras negativas em polaridade negativa e o licenciamento de um clítico que duplica um SN focalizado. PaLAVRas-Chave: sintaxe, Espanbol, clítico, foco. 


\section{Introducción.}

Este trabajo presenta un análisis generativo del clítico de Caso acusativo que duplica SSNN en una misma sentencia en español. Se demostrará que ese tipo de clítico puede dividirse en dos clases, la de los anafóricos y la de los deícticos. Se demostrará asimismo que la aparición de los últimos está vinculada a la focalización de un constituyente in situ. Este análisis se basa en la noción del foco relacional (Von Stechow 1986) y en los análisis de Aoun \& Li (1993) y Suñer (1995) que estudian elementos argumentales (a-barra) como fenómenos de polaridad.

En la sección 1 se discuten las principales teorías disponibles sobre el estatus de clítico. En la segunda sección se discute la cuestión del foco y se comentan clasificaciones recientes sobre los tipos de foco presentes en las lenguas. La sección 3 demuestra que, con base a Anderson (1993) se puede dividir dichos clíticos en dos clases, lo que se comprobará a través de juicios de gramaticalidad. En 4 se presenta el análisis de Suñer (1995) para la negación como fenómeno de polaridad negativa, y no de cuantificación negativa. En la sección 5 se demuestra el análisis generativo del doblado de clíticos como un fenómeno de polaridad, lo que garantiza la interpretación del un rasgo [ + foco] de un elemento in situ sin necesidad de recurso a movimiento en la Forma Lógica, estableciéndose un paralelo con el análisis de Suñer (1995) para la negación y de Aoun \& Li (1993) para palabras Wh-.

\section{La duplicación de clíticos.}

La llamada duplicación de clíticos en español corresponde formalmente a la aparición de elementos correferentes en la misma sentencia, el clítico y el SN. El SN dicho "duplicado" puede ser pleno o pronominal y la duplicación se da en regla variable. En tanto que la duplicación de SSNN de Caso dativo está extendida a todas las variedades del idioma, la duplicación de SSNN plenos de Caso acusativo puede observarse apenas en la variedad rioplatense del español, que comprende regiones de Argentina, Uruguay y Paraguay. El siguiente ejemplo, retirado de un corpus de español rioplatense, ejemplifica esa construcción:

(1) Yo la estudié esa esa cuestión ya, bastante. (Barrenechea:1982:460) 
En (1) el SN esa cuestión se presenta duplicado por un clítico la de Caso acusativo que guarda con éste coincidencia de rasgos de Caso, género y número. Además de considerada agramatical en otras zonas dialectales del español (andina, centroamericana y buena parte de la peninsular) esa duplicación, restricta a la lengua oral, presenta frecuencia de uso bastante menor que la que involucra Caso dativo ${ }^{1}$.

De otro lado, la duplicación de SSNN pronominales de Caso acusativo es obligatoria, no incurriendo, por lo tanto, en regla variable, y esa construcción se da en todas las variedades de la lengua.

(2a) La llamaron a ella. (Suñer, 1988:394)

(2b) * Llamaron a ella.

Hace falta notar la diferencia entre el doblado de clíticos en posición canónica y otra construcción común a todas variedades del español, que se da con el SN fuera de la posición canónica. Ese fenómeno se conoce por Clitic-Left dislocation (Cinque 1990).

(3a) *Ese libro, sólo encontré en la FNAC.

(3b) Ese libro, sólo lo encontré en la FNAC. (Raposo 2001:1)

Ese fenómeno está estrechamente vinculado a la codificación de la categoría discursiva tópico y en ese caso, la aparición del clítico preverbal se da de manera obligatoria, como lo demuestra la agramaticalidad del ejemplo (3b), siempre que haya un tópico en la sentencia ${ }^{2}$. La ausencia de un pronombre-copia del elemento topicalizado genera agramaticalidad en las lenguas que exhiben esa propiedad.

El clítico a analizarse en este trabajo es precisamente aquél ejemplificado en (1 a), que involucra duplicación de un SN pleno de Caso acusativo, nopronominal. Ese fenómeno como ya se dijo, se da en regla variable y es tema de gran controversia en la literatura generativista.

\footnotetext{
1 Un estudio variacionista realizado por Barrenechea \& Orecchia ha acusado una frecuencia de $8,6 \%$ de casos de duplicación de clíticos de acusativo, contra $68 \%$ de casos de duplicación con clíticos de Caso dativo. (Cf. Barrenechea \& Orecchia 1977).

2 Lo mismo no sucederá, sin embargo, en el caso de que el SN este bajo la acción de un foco. En ese caso, la sentencia no exhibirá el clítico, como se mostrará más adelante, en este trabalho.
} 


\subsection{Abordajes a la cuestión.}

En la literatura generativista que enfoca el fenómeno de los clíticos, éstos son vistos bajo diferentes enfoques, que se contradicen los unos a los otros. Kayne (1975) los analiza como argumentos que sufren movimiento. Se generarían en la posición argumental y luego, debido a su naturaleza clítica sufrirían extracción hacia dentro del núcleo verbal, lo que resulta en un núcleo verbal completo, que realiza posterior movimiento.

De otro lado, con base a una idea de Borer (1984), Jaeggli (1986b), haciendo uso de datos de duplicación de clíticos en español, desarrolla una hipótesis de estatus del clítico que contradice definitivamente la de Kayne. Inicialmente presentada en Jaeggli (1982), postula que los clíticos son elementos argumentales generados en la base, es decir, se generarían en la posición preverbal donde se superficializan. Dicha posición sería en núcleo de IP (la hipótesis se formuló, por lo tanto, antes de la propuesta de cisión de IP (Pollock 1989)). Allí se daría asimismo una adjunción con el verbo, en su monta hacia núcleos superiores. De acuerdo a ese abordaje, el clítico cobra propiedades argumentales a través de una cadena de coindexación con un pro pequeño en posición de argumento. Del mismo modo que Aoun (1981), Jaeggli asigna al elemento duplicado el estatus de adjunto.

En una propuesta más reciente, Franco (1993, 2000), basado en Suñer (1988) argumenta que los clíticos duplicantes, a pesar de generados en la base, no corresponden a argumentos, sino que constituyen un tipo de morfema de concordancia de objeto, fenómeno exhibido por lenguas como el vasco, por ejemplo.

Aquí se demostrará que los enfoques mencionados presentan problemas debido, precisamente, al hecho de tratar el clítico, desde el punto de vista sintáctico, como una categoría única. Como se demostrará a continuación, el rasgo [+/- nuevo] del SN duplicado (Prince, 1980), que corresponde al estatus informacional del $\mathrm{SN}$ se hace crucial en el juicio de gramaticalidad de las sentencias, lo que evidencia que el estatus de argumento o de adjunto del SN duplicado está vinculado a la diferencia [ + o - $]$ de ese rasgo. A esa diferencia en el status del SN corresponderá los diferentes estatus del clítico. 


\subsection{Dos tipos de clítico: anafórico y deíctico.}

Al trabajar con datos del corpus El Español Hablado en la Ciudad de Buenos Aires: Elementos para su Estudio (Barrenechea 1982), Correa (2003) demuestra la existencia de dos clíticos que duplican SSNN de Caso acusativo en español, lo que se demuestra en los ejemplos a continuación:

(4a) Yo la vi a Mercedes Sosa la primera vez que cantó. (Corpus:470)

(5a) La vi a la Q. D. fotografiada, y, qué gracioso, no se parece en nada a... (Corpus:369)

En cada uno de estos ejemplos, retirados del corpus, hay un SN pleno en posición canónica y un clítico en posición preverbal. En ambos casos, el clítico se refiere al complemento directo, corporificado por el SN. La diferencia cualitativa entre las sentencias estriba en el estatus informacional de cada uno de los SSNN, una información que, aunque va más allá de los límites de la sintaxis y trae información de orden pragmática al análisis, tiene reflejos en la interpretación y en el juicio de gramaticalidad de dichas sentencias.

Primer tipo: SN ya mencionado en el discurso.

(4a) Yo la vi a Mercedes Sosa la primera vez que cantó.

(4b) Yo la vi la primera vez que cantó.

Compárense (4a) y (4b). (4a) es una sentencia sacada del corpus y (4b) una alternativa con omisión del SN pleno. En (4b) el SN a Mercedes Sosa, de status informacional [-nuevo] pudo omitirse sin perjuicio de la interpretación ni de la gramaticalidad de la sentencia, lo que demuestra que dicho SN no constituye un argumento, sino más bien el adjunto denominado antitópico o afterthougt dentro de la terminología funcionalista.

Segundo tipo, clítico deíctico:

(5a) La vi a la Q. D. fotografiada, y, qué gracioso, no se parece en nada a...

(5b) * La vi y qué curioso, no se parece en nada a... 
En (5b), alternativa a la sentencia real (12a), el SN a la Q.D. fotografiada, de status informacional nuevo, por lo tanto, información nueva en el discurso, se omitió. El resultado fue perjuicio de la interpretación; la sentencia se ha vuelto agramatical.

El elemento que pudo omitirse, en este caso, es, precisamente, el clítico: Vi a la Q.D. fotografiada, y, qué gracioso, no se parece en nada a...

En esa diferencia estriba la clasificación propuesta en este trabajo: El clítico la del ejemplo (4a), al referirse a un ente viejo en el discurso, constituye el clítico anafórico ${ }^{3}$. Como el SN pudo omitirse, es un indicio de que el clítico está allí para la interpretación del objeto. Como tal, puede postularse que sea portador de papel temático y de Caso - conforme los tests sintácticos de (4) y (5) - y dichos rasgos pueden asignarse a elementos en posiciones argumentales. Estas observaciones están en total consonancia con lo previsto por Jaeggli (1982, 1986b).

En cambio, el clítico la en el ejemplo (5a), a pesar de exhibir la misma morfología, no encaja en la clasificación anterior. A este clítico se lo propone llamar clítico deíctico. La mención al clítico solo no sostiene la gramaticalidad de la sentencia, lo que puede indicar que no se trata del elemento responsable de la interpretación del objeto. En este caso, es el SN pleno el que porta los rasgos responsables por la interpretación del objeto. De ese modo, puede conjeturarse que el clítico deíctico no ocupa la misma posición estructural que el clítico anafórico, que se comporta en la forma prevista por Jaeggli $(1986 b)^{4}$.

\footnotetext{
3 Anafórico aquí debe entenderse como el clítico que retoma un referente presente en el discurso, en la configuración sintáctica presentada por Jaeggli $(1982,1986 b)$. No guarda relación con la noción de anáfora de los principios de vinculación.

4 Esta afirmación se basa en el trabajo de Anderson (1993), que compara la cliticización a la afijación: en tanto que la afijación se da entre un morfema y una raíz, la cliticización sería un tipo de afijación al nivel del sintagma. De ese modo, habría simetrías entre los afijos de palabra - los afijos propiamente dichos y los afijos de sintagma - los clíticos. Habría, entonces, clíticos flexionales y derivacionales, correspondiendo esta división a aquella que hay entre los afijos.

De ese modo, los clíticos flexionales "incluyen auxiliares, marcadores temporales y elementos pronominales representando los argumentos de una sentencia", (Anderson 1993:83), en tanto que a los clíticos derivacionales corresponde el contenido semántico o discursivo-funcional, "claramente distinto del tipo de material gramatical representado por el clítico flexional" (id.ibid.). La división entre clíticos anafóricos y deícticos, demostrada en esta sección ejemplifica claramente la división propuesta por el citado autor.
} 
Lo que parece estar en juego en el caso del español es una diferencia estructural dentro de una misma lengua, evidenciada por los diferentes juicios de gramaticalidad asignados a sentencias aparentemente iguales, diferencia que se pudo determinar por meio de un rasgo proveniente de la situación discursiva, en un nivel de interfaz de la Sintaxis con al pragmática. En tanto que los clíticos anafóricos son argumentales, y se vinculan a la idea de antitópico, habiendo sido explorados en los trabajos ya citados, queda por determinar las relaciones gramaticales de los clíticos deícticos, lo que se hará en el restante de este trabajo.

\section{E1 foco}

Como en este trabajo se demostrará que la aparición del clítico deíctico está ligada a la focalización de un componente in situ, esta sección describirá recientes abordajes a la cuestión del foco, así como una posible tipología de los focos en español.

Entre los recientes abordajes a la cuestión del foco descuellan el de Zubizarreta (1998) y el de Kiss (1998). Ambas autoras exploran las distinciones semánticas que presentan los focos. Zubizarreta divide los focos en dos tipos semánticos, foco no-contrastivo x foco contrastivo. De esa manera, el rasgo [contrastivo], como observado por Mioto (2003) es la base de la diferenciación semántica de los tipos de foco clasificados por esa autora.

Kiss (1998), por otro lado, basada en Szabolcsi (1981), además del rasgo [contrastivo], tiene en cuenta otro rasgo para efectuar su clasificación semántica de los tipos de foco. Este sería el rasgo [exaustividad]. Así, dentro de la clasificación semántica de los tipos de foco según Kiss (1998), se encuentran los focos:

a) informativo (wide focus);

b) contrastivo (narrow focus); $y$

c) de identificación exaustiva o exhaustivo (narrow focus).

De acuerdo a Kiss, a estos tres tipos de foco corresponden dos configuraciones sintagmáticas, la de foco in situ, para el tipo (a), y el foco estructural, para los tipos (b) y (c). 
En la siguiente muestra de habla, se pueden observar ejemplos de esas dos configuraciones sintácticas de foco:

(6) Enc. -Claro. ¿Pero tiene algún bobby especial, no? Inf. -No no.

(7) Enc. -HOBBIES ESPECIALES no tiene. Inf. -Eh... no no no no, bobbies especiales ninguno.

(Barrenechea 1982:36-37)

En ejemplo (6) demuestra el SN algún hobby especial bajo la acción de un foco informativo, en el que el elemento focalizado se mantiene en la posición en la que se ha generado. El ejemplo (7) demuestra el foco estructural, configuración del los tipos (b) y (c), en la que el elemento focalizado ocupa la periferia izquierda de la sentencia, o sea, se ha movido desde su posición inicial hasta una posición de escopo.

Hay que tener en cuenta que, en términos sintácticos, los focos de identificación exhaustiva y contrastivo no presentan diferencia, dentro de la tipología de Kiss.

Así, la clasificación de Kiss se diferencia de la de Zubizarreta pues, al incluir la noción de identificación exhaustiva, se crea una cisión dentro de la categoría foco estructural (Kiss 1995), aquel que ocupa la periferia izquierda.

La exhaustividad es una noción proveniente de trabajos ligados a la semántica formal, como el de Szabolcsi (1981)y supone la identificación de un elemento dentro de un conjunto dado por el contexto, como aclara Kiss:

"Un foco identificativo representa un subconjunto del conjunto de elementos dados situacionalmente o contextualmente presentes en el predicado; está identificado como el subconjunto exhaustivo de este conjunto presente efectivamente en el sintagma del predicado." Kiss (1998:249)

Es decir, al identificar un elemento de dado conjunto, el foco identificativo/exhaustivo pone en evidencia apenas ese elemento, descollado de un fondo que corresponde a los demás elementos del conjunto que no

5 Versión del original: "An identificational focus represents a subset of contextually or situationally given elements for which the predicate phrase can potentially hold; it is identified as the exhaustive subset of this set for which the predicate phrase actually holds." 
han sido focalizados. Kiss $(1995,1998)$ distingue claramente, en términos de estructura sintáctica, el foco de rasgo [-exhaustivo] de los focos de rasgo [+ exhaustivo]. El rasgo [+ exhaustivo] también distingue sintácticamente, para Kiss, los tipos de foco. En tanto que los focos dotados del rasgo $[+$ exhaustivo] (el contrastivo y el de identificación exaustiva, siempre en la concepción de Kiss) presentan la configuración de periferia izquierda, el foco [-exhaustivo] presenta la configuración in situ, posverbal.

La diferencia entre narrow focus y wide focus está estrictamente vinculada a ese rasgo. El primero, al ser [+exhaustivo] supone una interpretación relacional, o sea que ese foco tiene que interpretarse con la identificación de un elemento contra un fondo específico. El segundo, que caracteriza los elementos in situ, supone un elemento con el rasgo [-exhaustivo], cuya interpretación no se da de manera relativa, sino libre, sin necesidad de contraste con ningún otro elemento para obtenerse la interpretación adecuada. Kiss así resume la cuestión:

"Identificación exhaustiva puede expresarse solamente a través de un constituyente antepuesto en posición estructural de foco identificativo preverbal. Sin embargo, el constituyente cuyo único papel semántico-discursivo es el de marcar la novedad de la información que trae, no tiene posición marcada en la sentencia: éstos normalmente aparecen in situ en posición posverbal." ${ }^{6}$ Kiss (1998:249)

Este trabajo se servirá de la taxonomía de foco proveniente de Kiss (1998), en que pueden encontrarse en las lenguas naturales tres tipos de foco, por lo tanto: el contrastivo, el de identificación exhaustiva y el informativo.

En el análisis de las construcciones con clítico deíctico, se demostrará que, al contrario de Kiss, que prevé dos configuraciones sintácticas para tres tipos semánticos de foco diferentes, el español rioplatense presenta tres configuraciones estructurales distintas, precisamente correspondientes a los tres diferentes tipos de foco previstos por Kiss. Este análisis ha sido posible a través de una comparación entre el comportamiento del clítico y de la partícula negativa no en contextos de concordancia negativa en español.

\footnotetext{
6 Versión del original: "Exhaustive identification can be expressed only by a constituent preposed into the preverbal identificational focus slot. The constituents whose only semantic role is the marking of the novelty of the information they carry, however, have no distinguished position in the sentence; they tipically appear in situ in postverbal position."
} 


\section{La concordancia negativa como fenómeno de polaridad.}

Haegeman (1996) argumenta que el elemento negativo se implementa de manera semejante a la de Wh-, con base al criterio de operador (Rizzi, 1991, Haegeman, 1996). Eso significa decir el elemento dotado del rasgo $[+$ neg] tiene su interpretación vinculada a un operador generado en posición no-argumental (spec, $\mathrm{NegP}$ ), donde comparte rasgos de operador con el núcleo de ese sintagma.

En español, las sentencias abajo son agramaticales.

(8a) * Ella comió nada.

(8b) * Hablé con nadie.

Esto se debe a que el español exhibe el fenómeno de la polaridad negativa. Dentro de esa idea, un elemento [ + neg] posverbal sólo puede interpretarse adecuadamente en un contexto de concurrencia con otro elemento $[+$ neg]. Precisamente éste tiene que estar en posición preverbal, como lo demuestran los ejemplos a continuación:

(9a) Ella no comió nada.

(9b) No hablé con nadie.

Al observar las características de la polaridad negativa, Suñer (1995) propone que la sintaxis de la negación se dé en términos de polaridad negativa, y no como cuantificación negativa, como defiende Haegeman (1996). Dentro de esa visión, un elemento dotado del rasgo [ + neg] sólo puede licenciarse en una sentencia a través de una relación con un operador con el que comparta ese mismo rasgo (en este caso, $[+$ neg $]$ ) y cuya posición sea de escopo sobre el otro elemento de la polaridad. Es decir, el operador capaz de licenciar un elemento negativo / dotado de un rasgo [ + neg] debe compartir un rasgo de operador en una relación spec-head con un núcleo capaz de licenciar una posición no-argumental. Además, el operador c-comandará el elemento negativo en la sentencia. 
Una diferencia bastante común en la expresión de la negación entre variedades del español demuestra el operador negativo en uso:

(10a) No he ido nunca a ese sitio.

(10b) Nunca he ido a ese sitio.

Mientras (10a) es representativo de la negación en la modalidad peninsular del español, (10b) es una negación típica de la modalidad atlántica la lengua (variedad hablada en América). En este ejemplo, dentro de un contexto de polaridad negativa, ambos elementos $[+$ neg] tienen que licenciarse, tanto el elemento nunca, en posición posverbal, como la partícula no, en posición preverbal.

En la figura I, la representación sintáctica de (10a) el operador licencia sintácticamente el elemento negativo en la sentencia. De acuerdo a la teorización de Suñer (1995), en español, el núcleo Neg está ubicado entre [TP $\rfloor$ y $[\mathrm{AgrP}]$, del mismo modo que lo postulado para el italiano por Belletti (1990). Así, la sentencia (10a) tendría la siguiente representación (adaptada de Suñer, 1995:251):

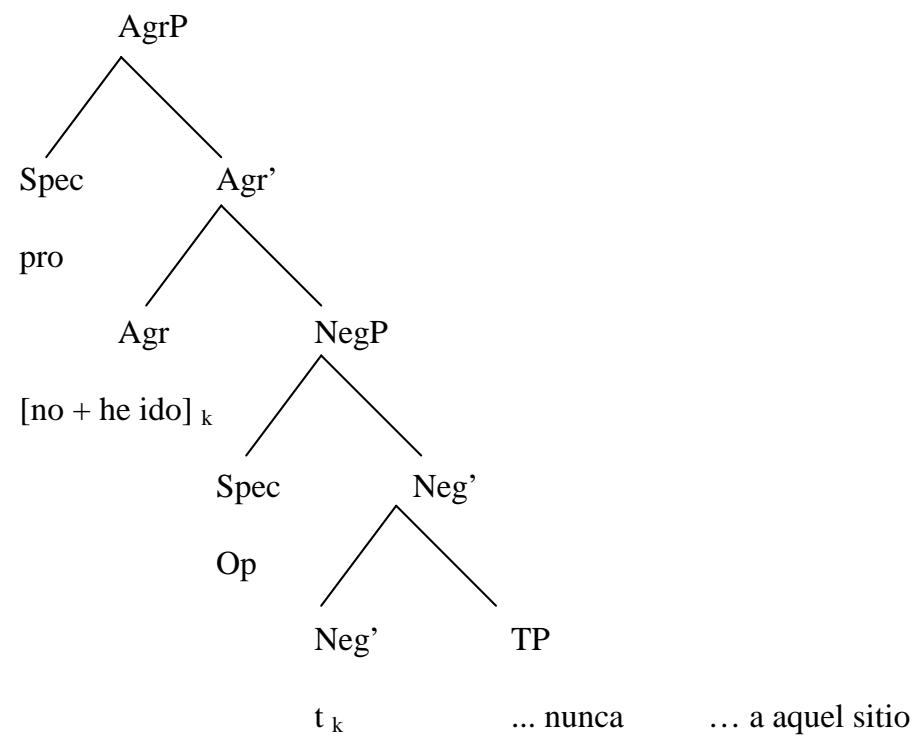

Figura I: No he ido nunca a aquel sitio 
Como elemento generado en [Spec, $\mathrm{NegP}$ ], en atención al criterio neg propuesto por Haegeman (1996) ${ }^{7}$, el operador negativo nulo puede ccomandar el elemento más bajo de la polaridad negativa, nunca. A su vez, el verbo, en su alzamiento de núcleo a núcleo hacia $[\mathrm{AgrP}]$, pasa por el núcleo $\mathrm{Neg}$, donde ha sido generado el segundo ítem de la polaridad negativa, el rasgo [+neg]. Se da la adjunción del rasgo $\{+$ neg $\}+$ verbo y ese núcleo complejo se mueve a $[\mathrm{AgrP}]$. Al salir del dominio del operador ([Spec, NegP]), el rasgo [ + neg] alzado por el verbo, deja de estar ccomandado por un elemento capaz de licenciarlo, ya que el operador habrá quedado en el subsintagma más bajo.

En este momento entra en operación la hipótesis de Suñer:

"El núcleo negativo necesita contenido fonético solamente si no está c-comandado por un elemento negativo" (Suñer, 1995:256)

De esa manera, el rasgo [ + neg] del núcleo negativo se lexicaliza (obtiene salida fonética) para que pueda licenciarse.

(11) *He ido nunca.

La agramaticalidad de este ejemplo puede explicarse por el hecho de que en elemento negativo nunca posverbal no mantiene relación de polaridad con otro elemento negativo no preverbal morfológicamente realizado. Si el operador negativo está ubicado en [Spec, NegP] (Belletti, 1990, Haegeman, 1996, Suñer, 1995), el núcleo Neg adjuntado al verbo en [Spec Agr] no está c-comandado por un operador que quedó en posición más baja. En este caso, el núcleo Neg, que porta un rasgo [ + neg] tendría que licenciarse a través de lexicalización. Como eso no sucede, resulta un elemento con un rasgo negativo que no habrá sido absorbido ni habrá obtenido salida fonética, lo que produce un crash en la derivación.

\footnotetext{
Haegeman (1996:144) propone un criterio neg- simétrico al criterio Wh de Rizzi (1991a:2):

Criterio NEG: a. Un operador-Neg debe estar en una configuración spec-head con un $\mathrm{X}^{\circ}$ con el rasgo [neg].

b. Un $\mathrm{X}^{\circ}$ con el rasgo [neg] debe estar en una configuración spec-head con un operador-Neg.

Dicha simetria conllevará u criterio unificado de operador (Kiss 1995, Rizzi 1997)
} 
La propuesta de Suñer es una alternativa a la propuesta de Haegeman (1996), que trata los elementos $[+$ neg] como cuantificadores, lo que hace que la interpretación de dichos elementos sea dependiente de movimiento de operador en la Forma Lógica. La propuesta de polaridad negativa pretende resolver la interpretación de esos elementos dentro de la misma Sintaxis, antes de la salida para la Forma Fonética, sin que haya, por lo tanto, que acudirse a movimiento posterior del operador, en la Forma Lógica, para obtenerse adecuadas relaciones de escopo.

Del mismo modo, también Aoun \& Li (1993) han propuesto un análisis de la sintaxis de elementos Wh- in situ en chino, basada en relaciones de polaridad, como alternativa al clásico trabajo de Huang (1982), que explica la interpretación de dichos Wh- a través de movimiento en Forma Lógica.

Suñer atribuye la polaridad negativa a todos tipos de negación en español. La negación en (10b), repetida aquí en la figura II, común en América, sin aparente polaridad negativa, se explica por el alzamiento del elemento negativo a la posición preverbal, como se puede observar en el diagrama, adaptado de Suñer (1995:253):

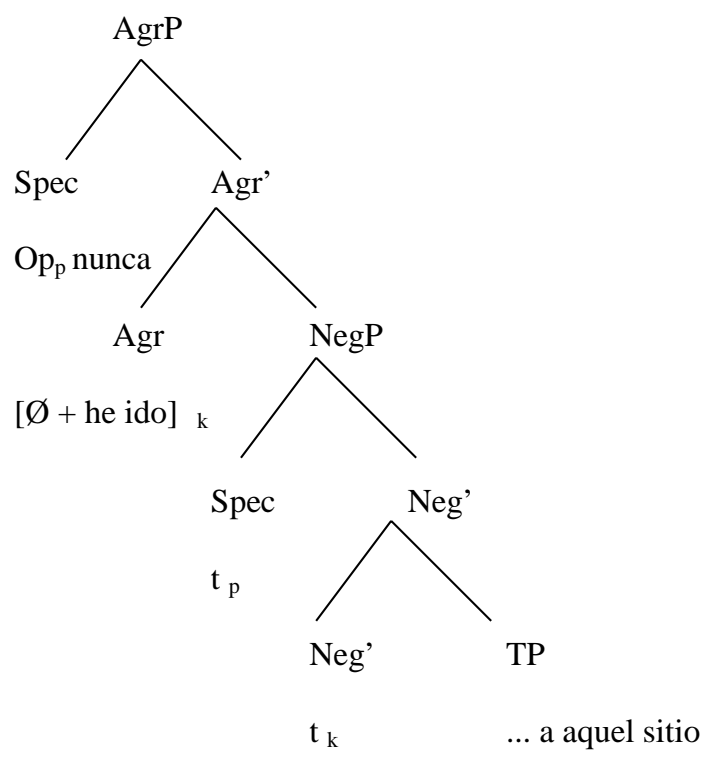

Figura II: Nunca he ido a aquel sitio 
Siempre de acuerdo con Suñer, el elemento negativo nunca esta vez ocupa [Spec, AgrP], que es la posición preverbal disponible en IP. Un elemento $[+$ neg] en posición preverbal necesita ser licenciado por un operador que pueda absorberle los rasgos. Sin embargo, el operador, desde la posición en la que se ha generado, no puede c-comandar un elemento [ + neg] preverbal. Para satisfacer el licenciamiento del elemento preverbal el operador nulo es atraído a la misma posición ocupada por el elemento movido. Allí hace una adjunción con nunca. El resultado de ese movimiento del operador nulo es que, desde la posición adonde fue movido, puede ccomandar y tener escopo sobre el elemento [+ neg] preverbal, de ahí resultando su licenciamiento sintáctico y su interpretación adecuada [+ negativo]. Con respecto al otro ítem de la polaridad, el rasgo [+ neg] generado en el núcleo negativo este sólo se lexicaliza si no está c-comandado por el operador. En este caso, no se lexicaliza, pues el operador nulo ya ccomanda y vincula la interpretación de los dos elementos de la polaridad. Eso impide la lexicalización del rasgo bajo la forma del clítico no, lo que atiende a un criterio de economía en la derivación. Eso puede comprobarse a través de (12).

(12) *Nunca no he ido a aquel sitio.

El ejemplo (12) demuestra la lexicalización del núcleo negativo cuando su interpretación ya está vinculada por el operador negativo nulo que lo ccomanda, lo que resulta en agramaticalicalidad. Si el movimiento de operador para obtener escopo sobre el elemento [+ neg] frontalizado se diera sólo en la Forma Lógica, el rasgo [+ neg], también fuera del escopo del operador, debido al movimiento verbal, tendría que lexicalizarse en Spell Out, lo que no sucede, como lo demuestra la agramaticalidad de (12).

De esa manera, Suñer establece tres condiciones para licenciamiento de ítemes negativos:

a. El licenciamiento de ítemes de polaridad negativa se obtiene a través del operador negativo nulo generado en Spec de Neg.

b. C-comando es el requisito estructural necesario para que el operador licencie el ítem de polaridad negativa;

c. Un elemento de polaridad negativa preverbal 'atrae' el operador debido a su rasgo formal $[+$ neg]; De esa manera, el alzamiento del operador es morfológicamente motivado. (Suñer, 1995:252). 
En términos interpretativos, la polaridad negativa demuestra un ejemplo de influjo de un elemento adyacente al verbo en la interpretación de un cuantificador o un indefinido ubicado bajo su escopo.

\section{El clítico deíctico como un focalizador.}

La cuestión del foco relacional puede relacionarse al tratamiento dado a la negación por Suñer. Bajo ese punto de vista, el foco en español también puede darse de dos maneras. A través del foco estructural (con el elemento focalizado en posición preverbal) o con el foco in situ (identificado por Kiss [1998], como el foco informativo). De acuerdo a lo expuesto, un elemento in situ puede ser adecuadamente interpretado de manera restrictiva sólo si está licenciado por un clítico verbal.

Del mismo modo que las sentencias nunca be ido a aquel sitio y no be ido nunca a aquel sitio son paráfrasis la una de la otra, la sentencia a continuación, con doblado de clítico, también podría parafrasearse:

\section{cl. deíctico SN nuevo[+ narrow focus]}

(13a) Yo la estudié esa cuestión ya, bastante. (Barrenechea, 1982:400)

$$
\text { [+ narrow focus] }
$$

(13b) ESA CUESTIÓN estudié yo, ya, bastante.

(13b) es una paráfrasis de (13a). Mientras (13b) está configurada dentro del foco estructural, (13a) marcaría un foco in situ en el SN, haciendo una identificación exhaustiva en el objeto, reforzada por el clítico la.

En (13a) en SN tiene una interpretación restricta, constituye la figura, contra un fondo constituido de otros elementos presupuestos - actualizados en la sentencia por el clítico - potencialmente involucrados en la acción denotada por el verbo (pudieran haberse estudiado). En una sentencia con el objeto in situ y sin el clítico, como (13c), el SN se interpreta libremente, sin que haya que tenerse en cuenta un fondo para su interpretación adecuada.

(13c) Yo ___ estudié esa cuestión ya, bastante. 
A esa construcción (13c) corresponde el foco informativo de Kiss (1998), caracterizado aquí como [-narrow focus]. Eso significa que este elemento entra en el llamado wide focus, que se interpreta sin necesidad de identificación exhaustiva o contraste.

Otra similitud que demuestra el funcionamiento de la polaridad es que del mismo modo que los elementos de polaridad $[+$ neg] cuando en posición preverbal no admiten el clítico no, los elementos $[+$ foco $]$ cuando en posición preverbal, no admiten el clítico lo/la:

(13d) *ESA CUESTIÓN la estudié yo ya, bastante. ${ }^{8}$

Siguiendo el criterio de operador unificado (Haegeman, 1996, Kiss, 1995, Rizzi, 1997), y el modelo de Suñer (1995) para la sintaxis de la negación como fenómeno de polaridad, se interpretan los focos [contrastivo] e [identificativo] (Kiss 1998) como fenómenos de polaridad. Estos focos son los que corresponden a la terminología generativista narrow focus. De otro lado el foco wide focus, correspondiente al foco informativo de Kiss no presenta relaciones de polaridad, innecesarias para su interpretación.

En la polaridad negativa el clítico no resulta ser una lexicalización del rasgo de operador [ + neg] del núcleo negativo. Este rasgo sólo se lexicaliza si no está c-comandado por un elemento correspondiente en la polaridad (un elemento negativo). En la propuesta de polaridad focal que se presenta en este trabajo, el clítico deíctico de Caso acusativo lo, la, los, las se analiza igualmente como una lexicalización del rasgo de operador que se hace presente siempre que el objeto correferente sea dotado de un rasgo [ + foco $]$ y éste no pueda c-comandar el núcleo de operador, ubicado en una proyección de IP. Si el objeto con un rasgo [ + foco $]$ está en posición de foco estructural (preverbal) no se licencia la lexicalización del rasgo [ + foco $]$ del núcleo de operador.

\footnotetext{
8 Hace falta notar aquí la diferencia entre foco y tópico, pues se admite el clítico resumptivo luego del elemento a la izquerda en las construcciones que involucran tópico, pero no las de foco, dentro de la idea de CLLD (Cinque, 1990).
} 
De ese modo, el ejemplo (13b) con el objeto con foco estructural, tendría la siguiente estructura, mostrada en la figura III:

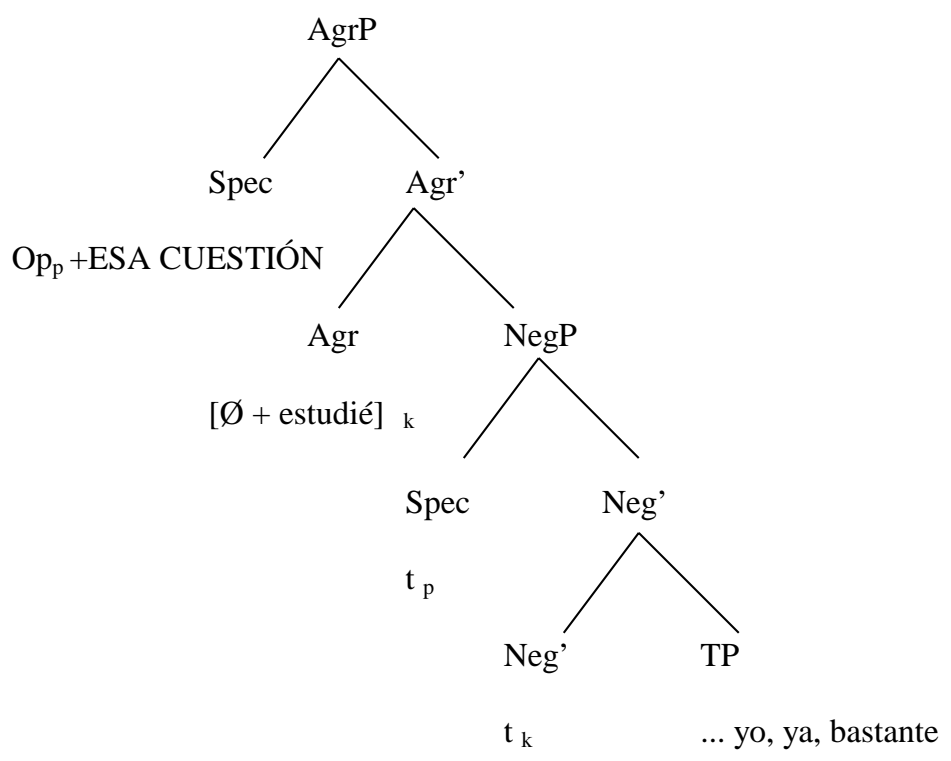

Figura III: ESA CUESTIÓN estudié yo ya, bastante

En esta representación, el SN [ + foco] ESA CUESTIÓN en posición preverbal atrae el operador nulo, que sale de $[\mathrm{Spec}, \mathrm{OpP}]$ y se adjunta al $\mathrm{SN}$ en [Spec, AgrP]. Desde esa posición, puede c-comandar el SN con el rasgo $[+$ foco $]$ y licencia su interpretación aún en la sintaxis. Del mismo modo, el otro ítem de la polaridad, el rasgo [ + foco], que tendrá que adjuntarse al verbo en su movimiento para chequeo de rasgos en $\mathrm{AgrP}$, por estar c-comandado por el operador, bajo su escopo, se neutraliza; su expresión ya no es necesaria, ni se desea por razones de economía.

El clítico deíctico constituye, así, un ítem de polaridad de operador, en este caso, de foco. Se manifiesta morfológicamente cuando el operador focal no tiene escopo sobre el rasgo, como en la figura IV, que representa el ejemplo (13a): 


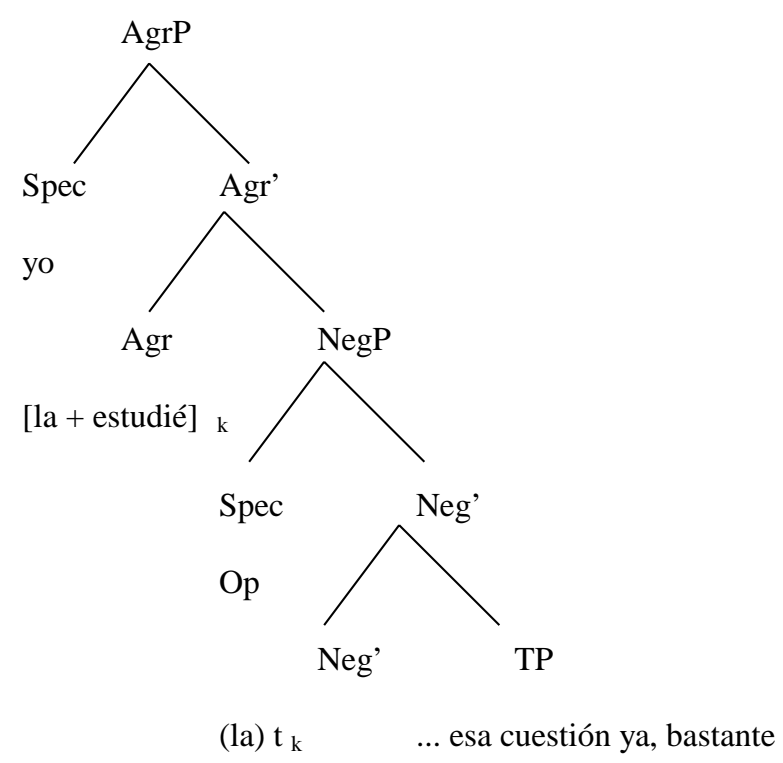

Figura IV: Yo la estudié esa cuestión ya, bastante

Como no hay movimiento del objeto focalizado para la posición preverbal, el operador focal nulo no tiene motivación para ocupar una posición más alta que el verbo y permanece donde fue generado. El rasgo generado en el núcleo de operador, alzado hasta el núcleo de Agr a través del movimiento verbal no ha sido absorbido y tiene que tener expresión fonética, lo que hace por medio de un clítico que suele mantener concordancia de género y número con el otro ítem de la polaridad focal. Como observado por Suñer (1995) y por Aoun \& Li (1993), el recurso a la polaridad resuelve la cuestión de la interpretación de operadores sin necesidad de acudir a movimiento en la Forma Lógica, recurso utilizado para explicar la interpretación de operadores cuando no se superficializan en posición de escopo sobre el verbo.

\section{Conclusión.}

Se ha buscado demostrar en este trabajo, a través de juicios de gramaticalidad y evidencias positivas identificadas en corpora naturales, 
que se puede, en efecto, asignar al español dos clases de clíticos de acusativo que duplican SSNN en posición canónica e que incurren en regla variable. Las teorías que buscan explicitar las relaciones gramaticales de los clíticos, aparentemente irreconciliables, cobran nuevo sentido a la luz de la dicotomía clítico anafórico x clítico deíctico. Del mismo modo, el trabajo demuestra el camino ineludible adonde convergen los estudios de la sintaxis formal, el de las interfaces con el Discurso y la Pragmática.

Se ha demostrado, asimismo, que el clítico deíctico puede estar vinculado a la expresión de foco en un elemento in situ, en un contexto de polaridad, lo que hace que el español rioplatense presente una posibilidad sintáctica extra de codificación del narrow focus no prevista por Kiss en su descripción del foco de identificación exhaustiva.

Recebido em fevereiro de 2004 Aprovado em novembro de 2005

E-mail: pacorrea@bol.com.br

\section{Referencias Bibliográficas}

Anderson, Stephen. 1993. Wackernagel's Revenge: Clitics, Morphology and the syntax of Second Position. Language 69: 68-89.

Aoun, Joseph. 1981. The formal nature of anaphoric relations. Doctoral dissertation, MIT.

\& Audrey Li. 1993. Wh- Elements in Situ: Syntax or LF? Linguistic Inquiry 24:199-238.

Barrenechea, Ana María. (org.) 1982. El Habla Culta de La Ciudad de Buenos Aires: Elementos para su Estudio. Buenos Aires: Universidad Nacional de Buenos Aires.

Borer, Hagit. 1984. Parametric Syntax: Case studies in semitic and romance languages. Dordrecht: Foris.

Cinque, Guglielmo. 1990. Types of A' Dependencies. Cambridge, Mass.: MIT Press.

Correa, Paulo. 2003. Clíticos que duplicam SN's de Caso acusativo em espanhol: Natureza, funções e representação sintática. Dissertação de Mestrado, Universidade Federal do Rio de Janeiro. 
Franco, Jon. 1993. On Object Agreement in Spanish. USC Doctoral Dissertation. Los Angeles, GSIL Publication Series. .2000. Agreement as a Continuum - the case of Spanish pronominal clitics. In:

Fritz Beukema \& M. den Dikken (eds.) Clitic Phenomena in European Languages. Amsterdam/Philadelphia: John Benjamins. Pp. 147-189. . \& Errapel Mejías-Bikandi. 1997. Overt and Covert Raising to [SpecAgrDO] and the Interpretation of Objects. Linguistic Analysis, 27: 1-2.

Haegeman, Liliane. 1996. The Typology of Syntactic Positions. L-relatedness and the A/A-bar distinction. In Wernwe Abraham et alli. Eds. Minimal ideas. Syntactic studies in the minimalist framework. Amsterdam and Philadelphia: John Benjamins.

HuANG, CTJ. 1982. Logical relations in Chinese \& theory of Grammar. Tesis doctoral, Massachusetts Institute of Technology.

Jaeggli, Osvaldo. 1982. Topics in Romance Syntax. Dordrecht: Foris. . 1986b. Three issues in the theory of clitics. In: Hagit Borer (ed.) The Syntax of Pronominal Clitics. (Syntax and Semantics, vol. 19). New York: Academic Press: 15-42.

KaYNe, Richard. (1975) French syntax. The transformational cycle. Cambridge, Mass.: MIT Press.

KIss, Katalin. 1995. Discourse Configurational Languages Introduction. In: K. Kiss (ed.) Discourse Configurational Languages. New York/ Oxford: Oxford University Press.

. 1998. Identificational focus versus Information Focus. Language 74, vol. 2 .

KrIfKA, Manfred. 1991. A Compositional Semantics for Multiple Focus Constructions, in Proceedings from Semantics and Linguistic Theory 1, Cornell Working Papers in Linguistics 10.

Mıтto, Carlos. 2000. CP no PB. Ms. Universidade Federal de Santa Catarina. . 2003a. Focalização e quantificação. Ms. Universidade Federal de Santa Catarina.

Prince, Ellen. 1980. On the Given/New Distinction. Ms. University of Pennsylvania.

Raposo, Eduardo. 2001. Objectos nulos e CLLD: uma teoria unificada. Ms. University of California at Santa Barbara. 
RizzI, Luigi. 1991 Residual Verb-second and the Wh- criterion. Technical Reports in Formal and Computational Linguistics 3. University of Geneva. . (1997) The Fine Structure of The Left Periphery. In: L. Haegeman, ed.. Elements of Grammar. Dordrecht: Kluwer, pp.281-337.

SzabolCSI, Anna. 1981. The semantics of topic-focus articulation. In: Jan Groenendijk et alli. (eds.) Formal methods in the study of language. Amsterdam: Matematisch Centrum: 513-541.

Von SteChow, Armin. 1991. Current Issues in the theory of Focus. Armin Von Stechow \& Dieter Wunderlich (eds.) Semantics. An International Handbook of Contemporary Research. Berlin: De Gruyter. . \& S. Uhmann. 1986. Some Remarks in Focus Projections. W. Abraham \& S. de Meij (eds.) Topic, Focus and Configurationality. Amsterdam: John Benjamins.

Suñer, Margarita. 1988. The role of agreement in clitic-doubled constructions. Natural Language and Linguistic Theory 6:391-434.

. 1995. Negative elements, island effects and resumptive no. The Linguistic Review 12: 233-273.

Zubizarreta, María-Luisa. 1998. Prosody, focus and word order. Cambridge, Mass.: MIT Press. 